\title{
Design of anisotropic composite shells using an isogeometric approach
}

\author{
Attila P. Nagy*, Mostafa M. Abdalla ${ }^{\dagger}$, Zafer Gürdal ${ }^{\ddagger}$ \\ Aerospace Structures, Delft University of Technology, Kluyverweg 1, 2629 HS Delft, The Netherlands
}

\begin{abstract}
Thin-walled composite structures, typically modeled as Cosserat continua during the design phase, are of particular importance in aerospace and automotive applications. At the dawn of industrial scale adoption of advanced fibre placement technology, it became viable to better exploit the directional properties of composite materials. In the recent past, numerous researches were devoted to the design of shells with optimal anisotropy. In the present work, combined stiffness tailoring and shape optimal design is proposed that is naturally facilitated in a non-uniform rational B-spline based isogeometric approach. Spatial variation of stiffness properties is parameterised by means of lamination parameters and the thickness of the shell. Shape changes are easily achieved by modifying selected control point co-ordinates and weights. The method of successive approximations has been employed to solve the optimisation problem. The formulation is separable in terms of sizing variables, however, separability of the shape design problem is not enforced. The design framework is verified through selected compliance minimisation problems.
\end{abstract}

\section{Introduction}

In the past decades, several studies have been dedicated to enhance the performance of laminated composite structures by tailoring local stiffness properties. Different design strategies have been developed that can be best classified as the way spatial variation of material anisotropy has been parameterised. As common feature of most methods, design is almost exclusively performed using an inextensible Cosserat shell theory with neglected shear flexibility, i.e. the classical lamination theory is invoked.

Direct parameterisation of fibre angle variation over the design domain was a natural choice as advanced fibre placement machines started to emerge. Fibre paths were typically assumed to take the form of (piecewise) continuous curves defined by a few parameters only. ${ }^{1-7}$ Reducing the set of design variables makes the approach particularly attractive for design optimisation. Continuity of the fibre courses are naturally preserved on the expense of a limited design space. It is noted however that an extension for non-developable surfaces is not straightforward, and applicability greatly depends on intrinsic surface measures.

Stiffness variation can also be defined as a function of local design variables defined per elements or nodes within a finite element context. Noting that the number of variables is related to the fidelity of the discretisation, employing local variables greatly extends the design space in general. Depending on the set of design variables two subclasses may be distinguished.

Design of variable stiffness panels using fibre angles as continuous variables that define the local stacking sequences was one of the earliest concepts. ${ }^{8-12}$ It is well known, however, that the local design problem is non-convex when paremeterised employing fibre orientations, which limits the use of traditional gradient based optimisers. Moreover, the number of variables increases proportionally with the number of layers in the stacking sequence. Preserving continuity of the fibre angles over the entire design domain compounds additional difficulties.

Alternatively, spatial variation of the stiffness properties may be defined by a finite set of continuous variables called lamination parameters. ${ }^{13-17}$ Employing lamination parameters is appealing from several

*PhD Student, Member AIAA. E-mail: a.p.nagy@tudelft.nl

${ }^{\dagger}$ Assistant Professor, Member AIAA.

${ }^{\ddagger}$ Professor, Aerospace Structures Chair, Delft University of Technology, Associate Fellow AIAA. 
perspectives. First of all, no explicit knowledge is required about the stacking sequence that obviates proliferation of local design variables. The feasible region in lamination parameter space is know to be convex, ${ }^{18}$ which is well suited for efficient gradient based optimisers. While the feasible space for membrane or bending only cases can be given analytically, ${ }^{19}$ currently no closed form solution for the combined feasible domain is known. Therefore it is typically approximated by a set of linear constraints obtained through successive convex hull approximations. ${ }^{20}$ The difficulty associated with the imposition of strength constraints has been recently alleviated by formulating a conservative strain based failure envelope. ${ }^{21}$ Optimal design obtained in lamination parameter space requires further post-processing, i.e. local stacking sequences need to be retrieved that also comply with known fabrication constraints. ${ }^{22}$

Essentially, structural sizing and shape optimisation can be easily coupled ${ }^{23}$ and this greatly extends the feasible design space. In the current work, a non-uniform rational B-spline (NURBS) based isogeometric design approach is proposed. It is intended to show that incorporating both sizing and shape design at an early design phase may substantially improve the performance of the final product.

The current work is built-up as follows. A short discussion of isogeometric analysis including the underlying geometric aspects is presented in section II. The design framework is introduced in III. The optimisation formulation is presented in section IV. The results of selected problems are discussed in section V and the work is concluded in section VI.

\section{Fundamentals of NURBS-based isogeometric analysis}

In the past two decades non-uniform rational B-splines inevitably became the standard apparatus for geometric representation in computer-aided design (CAD) ${ }^{24}$ Primarily for this reason, NURBS have been originally employed in isogeometric analysis, a novel branch of computational sciences. ${ }^{25}$ The approach represents a giant leap toward the synthesis between CAD and numerical analysis by alleviating the difficulties and inaccuracies primarily originating from the different geometric representations employed for design and analysis. ${ }^{26}$ More recent works unveiled several superior properties of the method over standard polynomial based finite element analysis in approximating the solution of a wide range of partial differential equations. ${ }^{27-30}$ The listed arguments make the isogeometric concept extremely attractive for design optimisation.

\section{II.A. Rational B-spline surfaces}

In general, a rational B-spline surface in the $d$-dimensional space is a piecewise rational surface composed as the linear combination of bivariate rational basis functions and the associated coefficients, commonly referred to as control points located in $\mathbb{R}^{d}$. Before giving the general formula of the surface however, some fundamental ideas are discussed.

First, the two-dimensional parametric space is introduced as the domain $\hat{\Omega}:=[0,1] \times[0,1]$. The parametric space is partitioned along both directions by means of knot vectors each comprising a set of non-decreasing real numbers called knots, i.e.

$$
\Xi=\left\{\xi_{1}=0, \ldots, \xi_{n+p+1}=1\right\}, \quad H=\left\{\eta_{1}=0, \ldots, \eta_{m+q+1}=1\right\},
$$

where $p, q, n$, and $m$ represent the degree and number of univariate spline basis functions defined on the corresponding knot vectors. Basically, knots play the role of co-ordinates in the parametric space. The characteristic intervals $\left[\xi_{i}, \xi_{i+1}\right) \times\left[\eta_{j}, \eta_{j+1}\right)$ and $\left[\xi_{1}, \xi_{n+p+1}\right] \times\left[\eta_{1}, \eta_{m+q+1}\right]$ are termed knot span and patch, respectively. Interior knots may be placed equidistantly or in a non-uniform fashion. While $p+1$ and $q+1$ multiplicity of the first and last knots yields open knot vectors and ensures endpoint interpolation of the surface, multiple interior knots reduce the continuity locally.

Given the knot vectors, the bivariate rational basis functions are furnished as

$$
R_{i, j}^{p, q}(\xi, \eta)=\frac{N_{i, p}(\xi) N_{j, q}(\eta) w_{i, j}}{\sum_{i=1}^{n} \sum_{j=1}^{m} N_{i, p}(\xi) N_{j, q}(\eta) w_{i, j}},
$$

where $N_{i, p}(\xi)$ and $N_{j, q}(\eta)$ are univariate spline basis functions defined recursively on $\Xi$ and $H$, respectively. ${ }^{24}$ The basis functions are non-negative, have a compact support, and constitute partition of unity at each parametric location. The symbol $w_{i, j}$ in equation (2) denotes the individual weights associated with each control points. 
Combining the control points $\mathbf{P}_{i, j}$ with the rational basis functions furnishes the piecewise smooth geometrical mapping ${ }^{27,31} \mathbf{F}: \hat{\Omega} \rightarrow \Omega$ connecting the parametric and physical spaces such that

$$
\mathbf{F}(\xi, \eta)=\sum_{i=1}^{n} \sum_{j=1}^{m} R_{i, j}^{p, q}(\xi, \eta) \mathbf{P}_{i, j}
$$

Basically, the geometrical mapping parameterises the physical domain. It has a piecewise smooth inverse, i.e. $\mathbf{F}^{-1}: \Omega \rightarrow \hat{\Omega}$, and both $\mathbf{F}$ and $\mathbf{F}^{-1}$ have non-singular Jacobians. The image of the geometrical mapping is a rational surface in $\mathbb{R}^{d}$, which is denoted by $\mathbf{r}$. The set of control points form an $n \times m$ control net. Setting all weights to unity naturally reduces equation (2) to bivariate spline basis functions and as a consequence the image of the mapping in equation (3) yields a B-spline surface.

Rational surfaces are often presented as the projected image of a B-spline surface in $\mathbb{R}^{d+1}$ defined by the control points of homogeneous form,

$$
\mathbf{P}_{i, j}^{w}=\left(\begin{array}{c}
\mathbf{P}_{i, j} w_{i, j} \\
w_{i, j}
\end{array}\right) .
$$

Essential geometric algorithms, e.g. knot refinement or order elevation, are performed on the homogeneous control net, which therefore plays a key role in generic computer-aided design.

\section{II.B. Basics of isogeometric shell analysis}

The isogeometric approach invokes the isoparametric concept, i.e. the solution of the physical problem is approximated using the basis functions used to describe the geometry. The NURBS space in $\Omega$ is given by the relation

$$
\mathcal{V}_{n, m}=\operatorname{span}\left\{R_{i, j}^{p, q} \circ \mathbf{F}^{-1}\right\}_{i=1, \ldots, n, j=1, \ldots, m},
$$

which is the push-forward of the NURBS space on the patch in $\hat{\Omega} \cdot{ }^{27}$ Conceptually, the numerical solution of a boundary value problem is sought in $\mathcal{V}_{n, m}$ such that given boundary conditions are satisfied.

Considering an arbitrary admissible variation of the shell's mid-surface $\delta \mathbf{r}$ and using the principal of virtual work, the static weak form of equilibrium is furnished as

$$
\delta W^{i}[\mathrm{~A}, \mathrm{~B}, \mathrm{D}, \epsilon(\mathbf{r}, \delta \mathbf{r}), \rho(\mathbf{r}, \delta \mathbf{r})]+\delta W^{e}=0,
$$

where $\delta W^{i}$ and $\delta W^{e}$ denote the virtual work of internal and external forces, respectively. The symbols A, B, and $\mathrm{D}$ stand for the forth order membrane, coupling, and bending stiffness tensors, respectively. The symbols $\epsilon$ and $\rho$ represent the relative membrane and bending strain measures, both being second order tensors. ${ }^{32}$ Following standard linearisation arguments, discretising equation (6), and invoking the isoparametric concept yields the well know consistent arrays used to approximate the structural response in a finite dimensional space defined in equation (5). ${ }^{31}$ For an elaborate discussion on the isogeometric formulation of the KirchoffLove shell element the reader is directed to the work of Kiendl et al. ${ }^{33}$

\section{Isogeometric shell design framework}

\section{III.A. Design parameterisation}

In the proposed isogeometric design framework, properties and/or design variables are naturally defined at the control points. While the set of shape design variables comprises selected control point co-ordinates and weights, the selection of sizing variables that reflect local changes in the stiffness requires somewhat more attention. Essentially, spatial variation of the stiffness over the design domain is uniquely defined by the set of twelve continuous parameters known as lamination parameters, ${ }^{34}$ and the shell's thickness. Considering symmetric laminates, i.e. $\mathrm{B}=0$, the number of lamination parameters is reduced to eight. Thus, in-plane and out-of-plane lamination parameters can be introduced as

$$
\begin{aligned}
\left(V_{1}, V_{2}, V_{3}, V_{4}\right) & =\int_{-1 / 2}^{1 / 2}(\cos 2 \theta(\bar{z}), \sin 2 \theta(\bar{z}), \cos 4 \theta(\bar{z}), \sin 4 \theta(\bar{z})) d \bar{z} \\
\left(W_{1}, W_{2}, W_{3}, W_{4}\right) & =12 \int_{-1 / 2}^{1 / 2} \bar{z}^{2}(\cos 2 \theta(\bar{z}), \sin 2 \theta(\bar{z}), \cos 4 \theta(\bar{z}), \sin 4 \theta(\bar{z})) d \bar{z}
\end{aligned}
$$


where $\bar{z}=z / h$ is the normalised through-the-thickness coordinate of the layers, ${ }^{35} h$ is the total thickness of the laminate, and $\theta(\bar{z})$ is the fibre angle at $\bar{z}$. Note that lamination parameters are related through the trigonometric functions used in their definition. At this point, the membrane and bending stiffness tensors can be defined as a linear function of the lamination parameters

$$
\begin{aligned}
& \mathrm{A}=h\left(\Gamma_{0}+V_{1} \Gamma_{1}+V_{2} \Gamma_{2}+V_{3} \Gamma_{3}+V_{4} \Gamma_{4}\right), \\
& \mathrm{D}=\frac{h^{3}}{12}\left(\Gamma_{0}+W_{1} \Gamma_{1}+W_{2} \Gamma_{2}+W_{3} \Gamma_{3} W_{4} \Gamma_{4}\right),
\end{aligned}
$$

where $\Gamma_{j}(j=0, \ldots, 4)$ are tensors in terms of laminate invariants. ${ }^{34,36}$ All tensors in equation (8) are in Cartesian basis. In the current work we further narrow our discussion to balanced laminates, i.e. $V_{i}=W_{i}=0$ with $i=2,4$. Hence, the total number of variables that uniquely define a laminate is reduced to five.

Variable stiffness designs are easily established by associating distinct stiffness tensors, as defined in equation (8), with the control points and interpolate from control points to the shell's mid-surface as detailed in section III.B. The general dependence of the constitutive law on the reference configuration is emphasized ${ }^{32}$ that $^{2}$ needs to be reflected once performing combined sizing and shape design.

\section{III.B. Interpolation schemes}

An important distinction is made between the mapping of typical properties and stiffness terms from control points contained in the convex hull to the parametric space. Evaluation of typical quantities, such as structural volume, requires regular spline interpolation to compute properties associated with a given parametric location that is written as

$$
b(\xi, \eta)=\sum_{k=i}^{i+p} \sum_{l=j}^{j+q} b_{k, l} R_{k, l}^{p, q}(\xi, \eta),
$$

where $i, j$, and $b$ denote the NURBS co-ordinates ${ }^{31}$ and an arbitrary property, respectively.

In order to ensure continuous variation of the resultant forces, the generic stiffness tensor C is interpolated in a inverse sense ${ }^{15,37}$ such that

$$
\mathrm{C}(\xi, \eta)=\left[\sum_{k=i}^{i+p} \sum_{l=j}^{j+q} \mathrm{C}_{k, l}^{-1} R_{k, l}^{p, q}(\xi, \eta)\right]^{-1},
$$

Note that the inverse of the stiffnes tensor is the compliance tensor, which gives equation (10) physical meaning. It is interesting to note that the delineated continuous sizing approach relies on the convex hull property of the underlying geometry, thereby ensuring the global stiffness matrix to remain positive definite during the sizing procedure.

\section{III.C. Multilevel design}

Despite the fact that sizing and shape optimal design may be combined in a straightforward fashion, it is essential to treat the two sets of design variables separately while aiming for an enhanced design strategy. Basically, increasing the number of sizing variables enables to distribute stiffness more effectively affecting the local stress field more accurately with a moderate increase in computational cost. On the contrary, enriching the shape design space and performing shape design and analysis at identical level of refinement may yield shapes that results in high local strain gradients, a generally undesired feature. In order to resolve this inherent difficulty a multilevel approach of narrow-tree form was proposed. ${ }^{37}$ Consequently, design variables sufficient to alter the shape at a reasonable fidelity are defined at a coarse grid, i.e. upper level, while sizing variables are defined in a more refined lower level model. Physical and sensitivity analyses of higher accuracy are performed at the refined, i.e. lower level model.

\section{Optimisation formulation}

In the present work, stiffness design, formulated as a compliance minimisation problem, of fibre-reinforced composite shells is considered. The compliance is computed as

$$
c=\frac{1}{2} \mathbf{f}^{T} \mathbf{u},
$$


where $\mathbf{f}$ and $\mathbf{u}$ stand for the external force vector and the linear displacement response, respectively. Sensitivity of the compliance with respect to an arbitrary design variable $x$ is given as

$$
\frac{\partial c}{\partial x}=\mathbf{u}^{T}\left[\frac{\partial \mathbf{f}}{\partial x}-\frac{1}{2} \frac{\partial \mathbf{K}}{\partial x} \mathbf{u}\right],
$$

where $\mathbf{K}$ denotes the linear stiffness matrix.

The optimisation problem is solved using successive approximations. Due to their computational efficiency, successive approximation methods are widely used in structural optimisation. ${ }^{38}$ The fundamental idea of the approach is to approximate both objective and constraint functions at a (feasible) design point. The approximations are typically constructed relying on zeroth and first order information. Optimisation is performed on the approximation leading to an updated design. This procedure is repeated until a converged solution is found. For separable problems, each term in the approximation depends on a subset of design variables associated with a control point in the isogeometric framework. Thus, the approximation subproblems can be efficiently solved in a parallel fashion using a dual approach. ${ }^{39}$

Considering stiffness tailoring, the compliance is approximated reciprocally and linearly in terms of the of the stiffness tensors and the thickness, respectively. The the general form of the separable approximation is furnished as

$$
c \approx \tilde{c}+\sum_{i \in \mathcal{S}}\left[\tilde{\Phi}_{i}^{m}:\left(\mathrm{A}_{i}^{-1}-\tilde{\mathrm{A}}_{i}^{-1}\right)+\tilde{\Phi}_{i}^{b}:\left(\mathrm{D}_{i}^{-1}-\tilde{\mathrm{D}}_{i}^{-1}\right)+\frac{\partial \tilde{c}}{\partial h_{i}}\left(h_{i}-\tilde{h}_{i}\right)\right]
$$

where $i$ sums over the set of control points $\mathcal{S}$ defined in the design formulation. The symbols $\tilde{\Phi}^{m}$ and $\tilde{\Phi}^{b}$ denote the compliance sensitivities with respect to the membrane and bending compliance, i.e. inverse stiffness tensors, respectively. The last term in equation (13) denotes explicit derivatives with respect to thickness, i.e. the thickness dependence of the compliance tensors is not taken into account here. Noting that the structural compliance is homogeneous of degree one in terms of both the compliance tensors and the thicknesses and by virtue of Euler's theorem for homogeneous functions, equation (13) can be rewritten

$$
c \approx \sum_{i \in \mathcal{S}}\left[\tilde{\Phi}_{i}^{m}: \mathrm{A}_{i}^{-1}+\tilde{\Phi}_{i}^{b}: \mathrm{D}_{i}^{-1}+\frac{\partial \tilde{c}}{\partial h_{i}} h_{i}\right]
$$

The approximation in equation (14) is convex, but not necessary conservative in general. To alleviate the issue of non-conservativeness a damping term is added to the formulation such that

$$
c \approx \sum_{i \in \mathcal{S}}\left[\tilde{\Phi}_{i}^{m}: \mathrm{A}_{i}^{-1}+\tilde{\Phi}_{i}^{b}: \mathrm{D}_{i}^{-1}+\frac{\partial \tilde{c}}{\partial h_{i}} h_{i}\right]+\bar{\rho}
$$

where $\bar{\rho}$ represents the damping. The overbar denotes that the damping term is sizing related. Generally, damping is a function of both exact and approximated function values and the design variables in the current and previous iterations, i.e. $\bar{\rho}:=f\left(V_{j}^{k}, V_{j}^{k-1}, W_{j}^{k}, W_{j}^{k-1}, c, \tilde{c}\right)$. Relevant constraint functions are also expanded similarly. It is worth highlighting that each function in the formulation has its own damping term. The damping values are updated in each iteration irrespective whether it is neglected due to nondecreasing objective function value or non-conservativeness of any functions. Thus, depending on previous iterates damping may be increased or decreased. The proposed method is basically a modified version of the conservative convex separable approximations originally proposed by Svanberg. ${ }^{40}$

Unlike for stiffness tailoring, the approximation employed for shape optimal design is non-separable and can be written as

$$
c \approx \tilde{c}+\frac{\partial \tilde{c}}{\partial s_{l}}\left(s_{l}-\tilde{s}_{l}\right)+\rho,
$$

where $s_{l}$ denotes selected control point co-ordinates. It is interesting to note that the price paid for this lack of separability is not too worrisome. This is due to the fact that shape design variables are typically orders of magnitude smaller than sizing ones. Similarly to sizing, a damping term is employed to ensure conservativeness of the design. An update procedure for the damping term has been implemented as well. For shape optimal design the damping does also depend on the shape change norm matrix, i.e. $\rho:=$ $f\left(\mathbf{H}, s_{l}^{k}, s_{l}^{k-1}, c, \tilde{c}\right)$. The shape change norm matrix has been originally introduced by Nagy et al. ${ }^{37}$ for 
isogeometric design of elastic arches. The shape change norm matrix for the current shell design problem is given as

$$
\mathbf{H}=\mathbf{K}\left(\mathrm{A}=\mathrm{I}, \mathrm{D}=d^{2} \cdot \mathbf{I}\right),
$$

where $d$ and $\mathrm{I}$ is the characteristic dimension of the geometry, e.g. the diameter of the sphere containing the patch(es), and the 3 by 3 identity tensor.

\section{Results}

\section{V.A. Problem statement}

Isogeometric design of a cantilever plate loaded at its tip is presented. The example problem is illustrated in Figure 1. Notice that the membrane only nature of the problem, which reduces the sizing design variables to the in-plane lamination parameters and the thicknesses. The initial geometry of the plate can be defined as a bilinear B-spline patch with control points at each corner of the plate. This representation, however, is not adequate for shape design and as a consequence moderate increase in the number of control points, i.e. shape design variables is required. Furthermore, the employed shell formulation is also in need of at least $\mathcal{C}^{2}$ spline basis functions, i.e. $p \geq 2$ and $q \geq 2$. To this end, the initial geometry has been $k$-refined ${ }^{26}$ and the biquadratic patch containing 4 by 1 knot spans was both utilised for shape design and also employed as the coarsest computational geometry. Additional multilevel design considerations, discussed in section III.C, require even finer meshes, which are easily obtained through subsequent $h$-refinement. The model utilised for shape design is depicted in Figure 2. The first and second principal material directions are defined along the $x$ and $y$ co-ordinate axis, respectively. For brevity, a single index numbering scheme for the control points has been introduced.

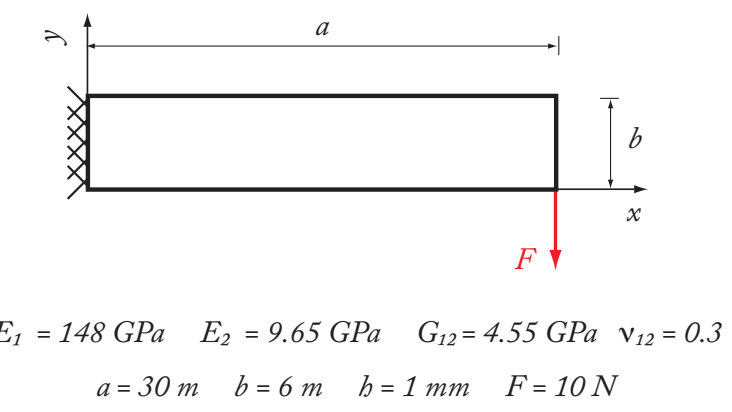

Figure 1. Schematic representation of the example problem

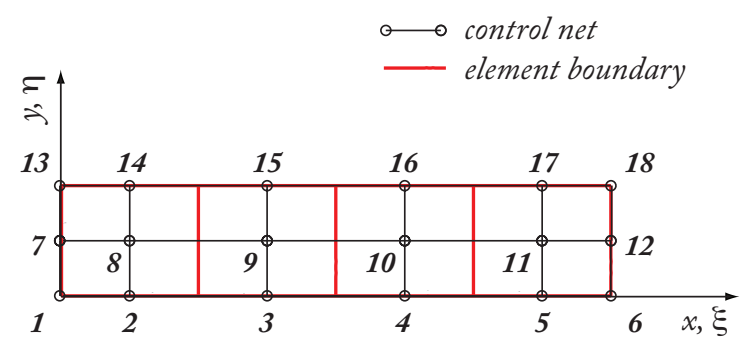

Figure 2. Shape design model and coarsest computational geometry

The example is solved in three different ways. First sizing for optimal anisotropy and shape optimal design will be considered separately, then their combination is discussed. The general form of the optimisation 
problem is written as

$$
\begin{array}{rl}
\min _{V_{j, i}, h_{i}, s_{l}} & c \\
\text { s.t. } & g_{l p} \leq 0 \\
& V=V_{0} \\
& h_{L} \leq h_{i} \leq h_{U} \\
& A \leq \frac{7}{10} \cdot 300 m^{2} \\
& 1.5 m \leq b \leq 10 m
\end{array}
$$

where $g_{l p}$ represents the feasibility constraints on lamination parameters, two for the current problem. ${ }^{19}$ The symbols $V, h_{L}, h_{U}$, and $A$ denote the volume, the lower and upper bounds on the thickness, and the area, respectively. The $i$ subscript ranges through all control points, i.e. $i=n \cdot m$. The zero subscript refers to the nominal design hereinafter. The nominal design is a quasi-isotropic isotropic laminate, i.e. $V_{j}=W_{j}=0$ $(j=1,3)$, with geometry and material properties as of Figure 1. Convergence criterion has been set on the objective function such that

$$
\frac{c^{k}-c^{k-1}}{c_{0}} \leq 10^{-3}
$$

Designing for optimal lamination parameter distribution relies on equations (18a) and (18b). Equations (18c) and (18d) are employed as the formulation is extended to include thickness design as well. In that case the volume constraint may be imposed as inequality.

Pure shape optimal design makes use of equations (18a), (18c), (18e), and (18f). The volume equality constraint in equation (18c) is used to solve for the thickness and explicitly eliminate it from the formulation. Thus, a single sizing variable is associated with all control points corresponding to a shell of uniform thickness. The optimiser, which relies on projected gradients, ${ }^{38}$ solves a problem that contains only shape design variables and the volume constraint is not included. Furthermore, notice that for the given load case the lower and upper bounds on the plate width, see equation (18f), will only be relevant for $y_{18}$ and $y_{13}$, respectively. With the same logic, any combination of sizing and shape design can be formulated in a straightforward manner.

\section{V.B. Design for optimal anisotropy}

A convergence study for optimal stiffness distribution is presented in Table 1. Each design was evaluated at the finest mesh containing 32 by 8 knot spans, i.e. elements $(e)$, and the compliance values were normalised with respect to the nominal quasi-isotropic laminate. While optimal constant stiffness (CS) design results in 48 percent improvement, the normalised compliance values is further diminished by an additional 7 percent for the variable stiffness (VS) case. It is interesting to note that all designs were obtained within 3 iterations. Optimal lamination parameter distributions are depicted in Figure 3. It is important to note that stiffer regions emerge along the top and the bottom edges of the plate. In those areas $V_{1}=V_{3}=1$, which correspond to fibers aligned in the $0^{\circ}$ direction, i.e. collinear to the $x$ co-ordinate axis. On the contrary, the middle of the plate contains softer regions of approximately quasi-isotropic stiffness properties.

\begin{tabular}{ccccc}
\hline & $e$ & design points & $c^{*} / c_{0}$ & iterations \\
\hline VS & $4 \times 1$ & $6 \times 3$ & 0.4737 & 3 \\
VS & $8 \times 2$ & $10 \times 4$ & 0.458 & 3 \\
VS & $16 \times 4$ & $18 \times 6$ & 0.4487 & 3 \\
VS & $32 \times 8$ & $34 \times 10$ & 0.4361 & 3 \\
$\mathrm{CS}$ & $32 \times 8$ & $1 \times 1$ & 0.5175 & 3 \\
\hline
\end{tabular}

Table 1. Numerical results for stiffness tailoring with lamination parameters as only design variables

Including thickness as design variable extends the feasible space and thereby allows further improvements, c.f. Table 2. For the current example the lower bound on the thickness was set to 10,30, 50, 70, and 90 percent of the nominal thickness, i.e. $h_{L}=i \cdot h_{0}$ with $i=0.1,0.3,0.5,0.7,0.9$. The upper bound was set 

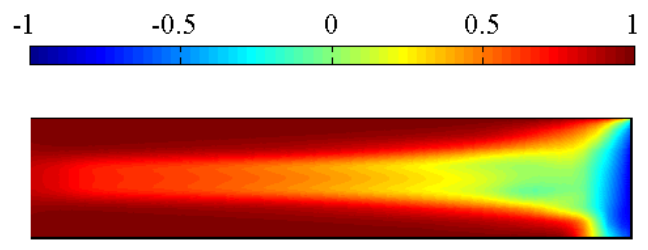

(a) $V_{1}$

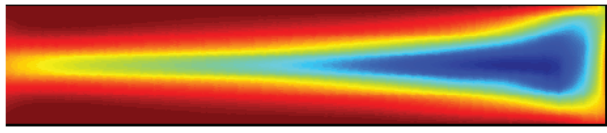

(b) $V_{3}$

Figure 3. In-plane lamination parameter distributions of the optimum variable stiffness plate using 34 by 10 design points

to ten times the nominal value, i.e. $h_{U}=10 \cdot h_{0}$. The results clearly demonstrate that the constraint on the lower bound always remains active and drives the design. Considering the investigated cases, decreasing the lower bound on the thickness may realise an up to 84 percent stiffer structure. Physical insight can be gained by looking at the optimal thickness distribution shown in Figure 4(c). Thickness is lumped along the bottom and top edges such that the final design is more efficient in balancing the in-plane reaction moment. The thickness build-up becomes more profound as moving closer to the support. Recalling a simple beam analogy, the final design may be viewed as an I-profile of continuously varying flange width correlating with the bending moment distribution along the $x$-axis. It is also interesting to note that the maximum thickness build-up converges to a plateau as the lower bound is decreased.

\begin{tabular}{ccccc}
\hline$h_{L} / h_{0}$ & $c^{*} / c_{0}$ & $h_{\min } / h_{0}$ & $h_{\max } / h_{0}$ & iterations \\
\hline 0.9 & 0.3583 & 0.9 & 2.9 & 4 \\
0.7 & 0.3008 & 0.7 & 4.3 & 4 \\
0.5 & 0.2742 & 0.5 & 5.5 & 5 \\
0.3 & 0.2641 & 0.3 & 6.3 & 6 \\
0.1 & 0.2635 & 0.1 & 6.5 & 6 \\
\hline
\end{tabular}

Table 2. Stiffness tailoring results considering both lamination parameters and thickness as design variables on 34 by 10 design points

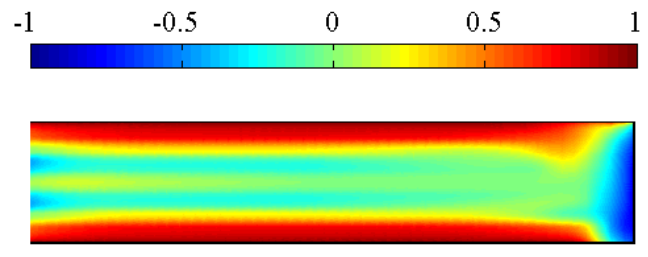

(a) $V_{1}$

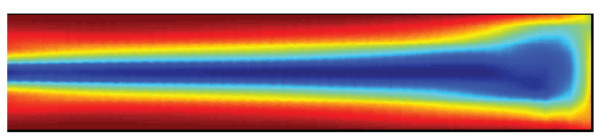

(b) $V_{3}$

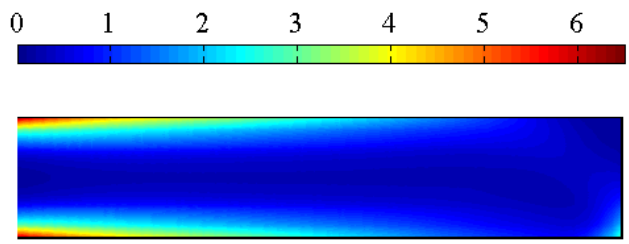

(c) $h / h_{0}$

Figure 4. In-plane lamination parameter and thickness distributions of the optimum variable stiffness and thickness plate using 34 by 10 design points and setting $h_{L}=0.1 \cdot h_{0}$ 


\section{V.C. Shape optimal design}

Three cases has been distinguished and investigated when performing shape design of the cantilever plate.

Case 1:

Utilising the single index notation of the control points in Figure 2, the set of design variables comprises the $y$ co-ordinates of the control points along $y=b$, i.e. $s_{l}=y_{i}$ with $i=13, \ldots, 18$. The $y$ co-ordinates of the control points at $y=b / 2$ are related to the design variables such that $y_{j}=0.5 \cdot y_{i}$ with $j=7, \ldots, 12$. Strictly speaking the current problem is more sizing alike in the sense that the optimal design variables will directly reflect the bending moment distribution along the $x$ axis.

Shape optimal design of the current case with isotropic material properties has been presented by Wall et $a l .{ }^{41}$ Also, no volume constraint has been imposed in the cited work. In the current problem formulation, however, the volume constraint plays an essential role by providing a fair basis of comparison between the sized only and shape optimal designs.

\section{Case 2:}

The set of design variables contains the $y$ co-ordinates of the control points along $y=b$ and $y=b / 2$, i.e. $s_{l}=y_{i}$ with $i=7, \ldots, 18$. In order to overcome difficulties associated with the concentrated force, an additional constraint has been imposed such that $y_{12} \leq 0.5 \cdot y_{18}$. In contrast to Case 1, a multilevel design scheme has been utilised. Finer meshes used to evaluate function values and sensitivities comprised 8 by 2 , 16 by 4 , and 32 by 8 knot spans.

Case 3:

This is the most general shape design case considered that includes the design variables $s_{l}=\left(x_{i}, y_{j}\right)$ with $i=8, \ldots, 11,14, \ldots, 17$ and $j=7, \ldots, 18$. Similarly to Case 2, an additional tip constraint was introduced and the multilevel design scheme has been utilised.

The numerical results are listed in Table 3. It is emphasized that the number of elements $(e)$ in the second column refer to the mesh at which physical and sensitivity analyses were performed. In the iterations column, the numbers in parentheses indicate the neglected iterations. The total number of iterations never exceeded 13. The results show around 53 percent decrease in compliance. For Case 2 and Case 3 minor improvements are accounted for the increasing fidelity of the analysis grid utilised in the multilevel scheme. Maintaining the initial volume resulted about 15 percent decrease in the thickness of the shape optimal plate. Optimal shapes are depicted in Figure 5. Corresponding convergence charts for both the function values and the damping terms are illustrated in Figure 6.

\begin{tabular}{ccccc}
\hline & $e$ & $c^{*} / c_{0}$ & $h / h_{0}$ & iterations \\
\hline Case 1 & $4 \times 1$ & 0.4707 & 0.8572 & $10(2)$ \\
Case 2 & $8 \times 2$ & 0.4704 & 0.8572 & $8(4)$ \\
& $16 \times 4$ & 0.47 & 0.8572 & $8(4)$ \\
& $32 \times 8$ & 0.4699 & 0.8572 & $8(4)$ \\
Case 3 & $8 \times 2$ & 0.4688 & 0.8572 & $10(3)$ \\
& $16 \times 4$ & 0.4684 & 0.8572 & $10(3)$ \\
& $32 \times 8$ & 0.4684 & 0.8572 & $9(3)$ \\
\hline
\end{tabular}

Table 3. Shape optimisation results

\section{V.D. Combined sizing and shape design}

At last stiffness tailoring of the shape optimal design attained for Case 3 is performed. The results for the different sets of design variables are presented in Tables 4 and 5. In comparison to the nominal design, combined shape and stiffness design yields 75 and 80 percent stiffer designs considering constant and variable stiffness laminates, respectively. Designing the thickness pattern contributes to an at most 5 percent additional improvement. Optimal lamination parameter and thickness distributions are depicted in Figures 7 and 8. 


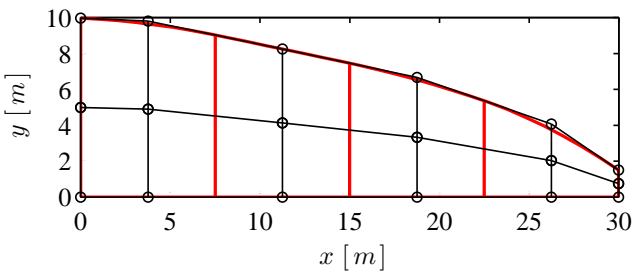

(a) Case 1

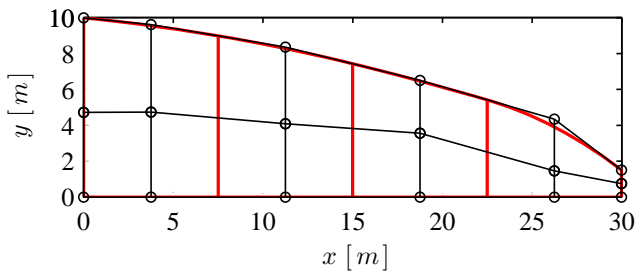

(b) Case 2

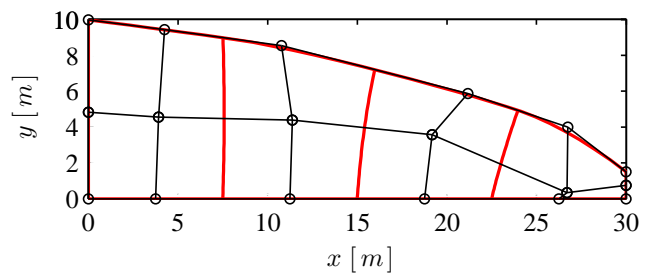

(c) Case 3

Figure 5. Optimal shapes obtained in the different design cases

\begin{tabular}{ccccc}
\hline & $e$ & design points & $c^{*} / c_{0}$ & iterations \\
\hline $\mathrm{VS}$ & $32 \times 8$ & $34 \times 10$ & 0.2054 & 3 \\
$\mathrm{CS}$ & $32 \times 8$ & $34 \times 10$ & 0.2521 & 2 \\
\hline
\end{tabular}

Table 4. Numerical results for combined shape design and stiffness tailoring with lamination parameters as only sizing variables

\begin{tabular}{ccccc}
\hline$h_{L} / h_{0}$ & $c^{*} / c_{0}$ & $h_{\min } / h_{0}$ & $h_{\max } / h_{0}$ & iterations \\
\hline 0.9 & 0.1840 & 0.9 & 4.5499 & 3 \\
0.7 & 0.1639 & 0.7 & 5.4832 & 4 \\
0.5 & 0.1512 & 0.5 & 6.1832 & 5 \\
0.3 & 0.1444 & 0.3 & 6.6499 & 6 \\
0.1 & 0.1428 & 0.1 & 6.8832 & 6 \\
\hline
\end{tabular}

Table 5. Numerical results for combined shape design and stiffness tailoring with both lamination parameters and thickness as sizing variables

\section{Conclusions}

A NURBS based isogeometric design environment for thin-walled composite shells has been proposed. The framework integrates both stiffness tailoring, i.e. structural sizing, and shape optimal design. While the stiffness design was parameterised in terms of lamination parameters and thickness values defined control point-wise, the set of shape variables naturally comprised selected control point co-ordinates. The optimisation problem has been solved using the method of successive approximations. The different nature of the constructed approximations is worth highlighting. Stiffness tailoring makes use of a separable approximation defined in terms of the compliance tensors and the thickness. In contrast, separability has not been enforced for shape design, and the final form of the approximation is furnished in a simple linear expansion in terms of shape variables. The approach has been verified on compliance minimisation problems. Current work investigates to couple sizing and shape design and perform them in a simultaneous fashion. 


\section{References}

${ }^{1}$ Gürdal, Z. and Olmedo, R., "Composite laminates with spatially varying fiber orientations - Variable stiffness panel concept," NASA Technical Report No. 19920051737, Tech. rep., 1992.

${ }^{2}$ Olmedo, R. and Gürdal, Z., "Buckling response of laminates with spatially varying fiber orientations," Proceedings of the 34th AIAA/ASME/ASCE/AHS/ASC Structures, Structural Dynamics and Materials Conference, 1993.

${ }^{3}$ Nagendra, S., Kodiyalam, A., Davis, J. E., and Parthasarathy, V. N., "Optimization of tow fiber paths for composite design," Proceedings of the 36th AIAA/ASME/ASCE/AHS/ASC Structures, Structural Dynamics and Materials Conference, 1995.

${ }^{4}$ Parnas, L., Oral, S., and Ceyhan, Ü., "Optimum design of composite structures with curved fiber courses," Composites Science and Technology, Vol. 63, No. 7, 2003, pp. 1071-1082.

${ }^{5}$ Gürdal, Z. and Tatting, B. F., "Tow-placement technology and fabrication issues for laminated composite structures," Proceedings of the 46th AIAA/ASME/ASCE/AHS/ASC Structures, Structural Dynamics and Materials Conference, 2005.

${ }^{6}$ Blom, A. W., Setoodeh, S., Hol, J. M. A. M., and Gürdal, Z., "Design of variable-stiffness conical shells for maximum fundamental eigenfrequency," Computers 83 Structures, Vol. 86, 2008, pp. 870-878.

${ }^{7}$ Alhajahmad, A., Abdalla, M. M., and Gürdal, Z., "Design tailoring for pressure pillowing using tow-placed steered fibers," Journal of Aircraft, Vol. 45, No. 2, 2008, pp. 630-640.

${ }^{8}$ Hyer, M. W. and Charette, R. F., "The use of curvilinear fiber format in composite structure design," Proceedings of the 30th AIAA/ASME/ASCE/AHS/ASC Structures, Structural Dynamics and Materials Conference, 1989.

${ }^{9}$ Hyer, M. W. and Lee, H. H., "The use of curvilinear fiber format to improve buckling resistance of composite plates with central circular holes," Computers \&5 Structures, Vol. 18, 1991, pp. 239-261.

${ }^{10}$ Pedersen, P., "Examples of density, orientation, and shape-optimal 2D-design for stiffness and/or strength with orthotropic materials," Structural and Multidisciplinary Optimization, Vol. 26, 2004, pp. 37-49.

${ }^{11}$ Huang, J. and Haftka, R. T., "Optimization of fiber orientations near a hole for increased load-carrying capacity of composite laminates," Structural and Multidisciplinary Optimization, Vol. 30, No. 5, 2005, pp. 335-341.

${ }^{12}$ Setoodeh, S., Gürdal, Z., and Watson, L. T., "Design of variable-stiffness composite layers using cellular automata," Computer Methods in Applied Mechanics and Engineering, Vol. 195, 2006, pp. 836-851.

${ }^{13}$ Setoodeh, S., Abdalla, M. M., Gürdal, Z., and Tatting, B. F., "Design of variable-stiffness composite laminates for maximum in-plane stiffness using lamination parameters," 2005.

${ }^{14}$ Setoodeh, S., Abdalla, M. M., and Gürdal, Z., "Design of variable stiffness laminates using lamination parameters," Composites Part B, Vol. 37, 2006, pp. 301-309.

${ }^{15}$ Abdalla, M. M., Setoodeh, S., and Gürdal, Z., "Design of variable stiffness composite panels for maximum fundamental frequency using lamination parameters," Composite Structures, Vol. 81, No. 2, 2007, pp. 283-291.

${ }^{16}$ IJsselmuiden, S. T., Abdalla, M. M., and Gürdal, Z., "Thickness tailoring of variable stiffness panels for maximum buckling load," Proceddeigns of the 17th International Conference on Composite Materials, Apr 2009, pp. 1-10.

${ }^{17}$ IJsselmuiden, S. T., Abdalla, M. M., and Gürdal, Z., "Optimization of variable-stiffness panels for maximum buckling load using lamination parameters," AIAA Journal, Jan 2010.

${ }^{18}$ Hammer, V. B., Bendsøe, M. P., Lipton, R., and Pedersen, P., "Parametrization in laminate design for optimal compliance," International Journal of Solids and Structures, Vol. 34, No. 4, 1997, pp. 415-434.

${ }^{19}$ Miki, M. and Sugiyama, Y., "Optimum design of laminated composite plates using lamination parameters," $A I A A$ Journal, Vol. 31, No. 5, Jan 1993, pp. 921-922.

${ }^{20}$ Setoodeh, S., Abdalla, M. M., IJsselmuiden, S. T., and Gürdal, Z., "Design of variable-stiffness composite panels for maximum buckling load," Composite Structures, Vol. 87, 2009, pp. 109-117.

${ }^{21}$ IJsselmuiden, S. T., Abdalla, M. M., and Gürdal, Z., "Implementation of strength-based failure criteria in the lamination parameter design space," AIAA Journal, Vol. 46, No. 7, Jun 2008, pp. 1826-1834.

${ }^{22}$ Setoodeh, S. and Blom, A. W., "Generating curvilinear fiber paths from lamination parameters distribution," Proceedings of the 47th AIAA/ASME/ASCE/AHS/ASC Structures, Structural Dynamics and Materials Conference, 2006.

${ }^{23}$ Zhou, M., Pagaldipti, N., Thomas, H. L., and Shyy, Y. K., "An integrated approach to topology, sizing and shape optimization," Structural and Multidisciplinary Optimization, Vol. 26, 2004, pp. 308-317.

${ }^{24}$ Piegl, L. and Tiller, W., The NURBS book - Monographs in visual communication, Springer-Verlag Publishing Company, Inc., Heidelberg, 2nd ed., 1995.

${ }^{25}$ Hughes, T. J. R., Cottrell, J. A., and Bazilevs, Y., "Isogeometric analysis: CAD, finite elements, NURBS, exact geometry and mesh refinement," Computer Methods in Applied Mechanics and Engineering, Vol. 194, 2005, pp. 4135-4195.

${ }^{26}$ Cottrell, J. A., Hughes, T. J. R., and Reali, A., "Studies of refinement and continuity in isogeometric structural analysis," Computer Methods in Applied Mechanics and Engineering, Vol. 196, 2007, pp. 4160-4183.

${ }^{27}$ Bazilevs, Y., da Veiga, L. B., Cottrell, J. A., Hughes, T. J. R., and Sangalli, G., "Isogeometric analysis: Approximation, stability and error estimates for $h$-refined meshes," Mathematical Models and Methods in Applied Sciences, Vol. 16, No. 7, Feb 2006, pp. 1031-1090.

${ }^{28}$ Cottrell, J. A., Reali, A., Bazilevs, Y., and Hughes, T. J. R., "Isogeometric analysis of structural vibrations," Computer Methods in Applied Mechanics and Engineering, Vol. 195, 2006, pp. 5257-5296.

${ }^{29}$ Hughes, T. J. R., Reali, A., and Sangalli, G., "Duality and unified analysis of discrete approximations in structural dynamics and wave propagation: comparison of $p$-method finite elements with $k$-method NURBS," Computer Methods in Applied Mechanics and Engineering, Vol. 197, No. 49-50, 2008, pp. 4104-4124.

${ }^{30}$ Evans, J., Bazilevs, Y., Babuška, I., and Hughes, T., "n-Widths, sup-infs, and optimality ratios for the k-version of the isogeometric finite element method," Computer Methods in Applied Mechanics and Engineering, Vol. 198, No. 21-26, Jan 2009, pp. $1726-1741$. 
${ }^{31}$ Cottrell, J. A., Hughes, T. J. R., and Bazilevs, Y., Isogeometric analysis: Toward the integration of CAD and FEA, John Wiley \& Sons Ltd., Singapore, 2009.

${ }^{32}$ Simo, J. C., "A finite strain beam formulation. The three-dimensional dynamic problem. Part I," Computer Methods in Applied Mechanics and Engineering, Vol. 49, 1984, pp. 55-70.

${ }^{33}$ Kiendl, J., Bletzinger, K.-U., Linhard, J., and Wüchner, R., "Isogeometric shell analysis with Kirchhoff-Love elements," Computer Methods in Applied Mechanics and Engineering, Vol. 198, No. 49-52, Jan 2009, pp. 3902-3914.

${ }^{34}$ Tsai, S. W. and Hahn, H. T., "Introduction of composite materials," Technomic Publishing Company, Inc., Vol. Lancaster, 1980.

${ }^{35}$ Gürdal, Z., Haftka, R. T., and Hajela, P., Design and optimization of laminated composite materials, John Wiley \& Sons Ltd., New York, 1999.

${ }^{36}$ Jones, R. M., Mechanics of composite materials, Taylor and Francis, Inc, Washington, D.C., 2nd ed., 1998.

${ }^{37}$ Nagy, A. P., Abdalla, M. M., and Gürdal, Z., "Isogeometric sizing and shape optimisation of beam structures," Computer Methods in Applied Mechanics and Engineering, Vol. 199, Jan 2010, pp. 1216-1230.

${ }^{38}$ Haftka, R. T. and Gürdal, Z., Elements of structural optimization, Kluwer Academic Publishers, Dordrecht, 1992.

${ }^{39}$ Fleury, C. and Schmit, L. A., "Dual methods and approximation concepts in structural synthesis," NASA Contractor Report 3226, Jul 1980, pp. 1-225.

${ }^{40}$ Svanberg, K., "A class of globally convergent optimization methods based on conservative complex separable approximations," SIAM Journal on Optimization, Vol. 12, No. 2, 2002, pp. 555-573.

${ }^{41}$ Wall, W. A., Frenzel, M. A., and Cyron, C., "Isogeometric structural shape optimization," Computer Methods in Applied Mechanics and Engineering, Vol. 197, 2008, pp. 2976-2988. 


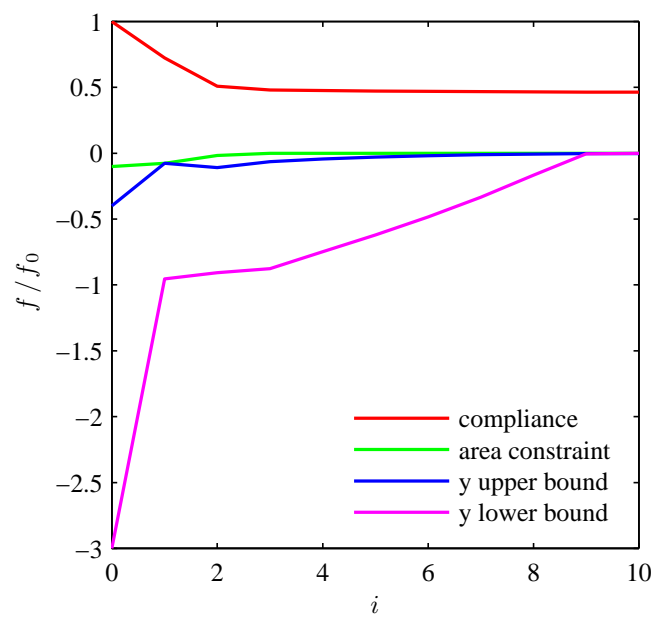

(a) Case 1

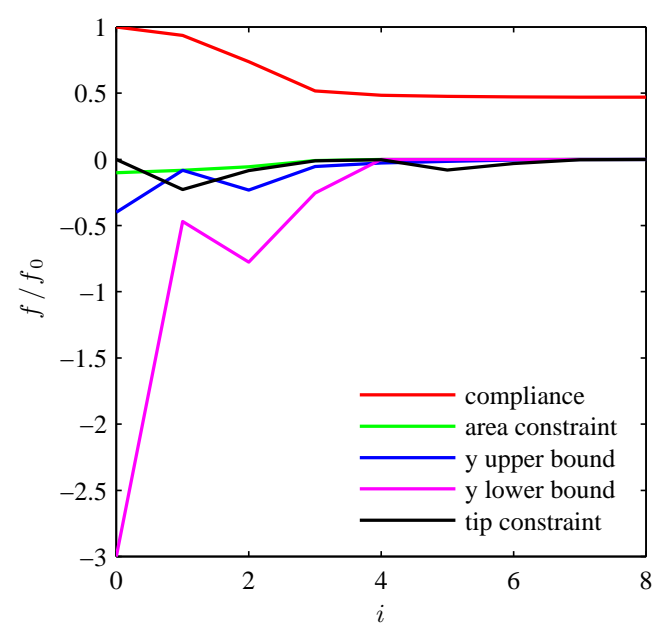

(c) Case 2

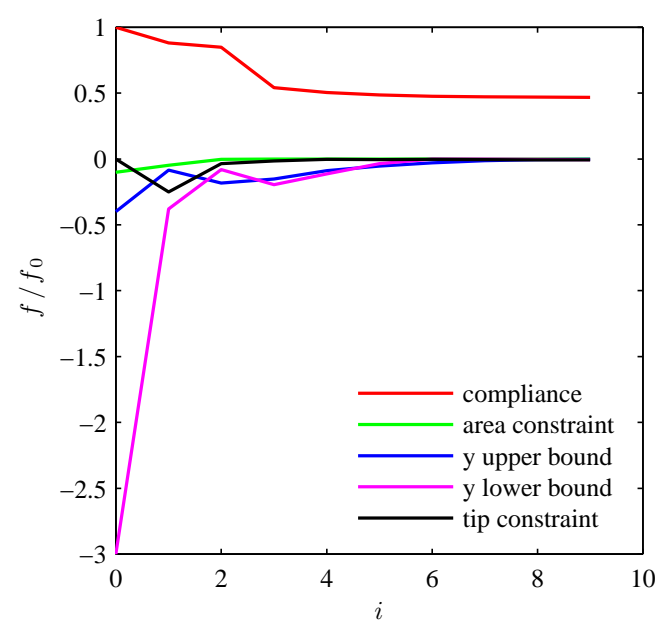

(e) Case 3

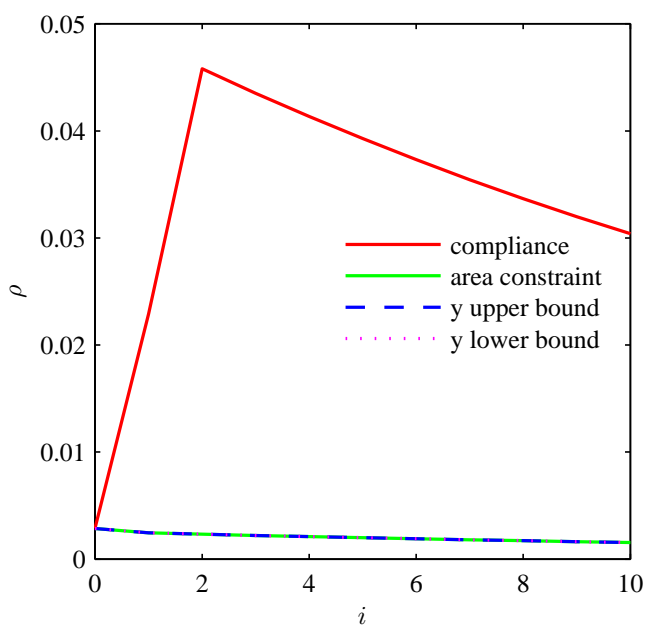

(b) Case 1

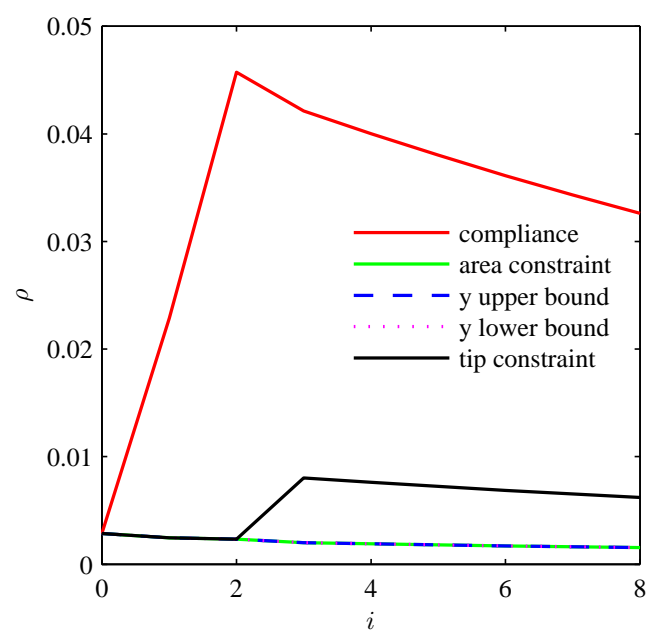

(d) Case 2

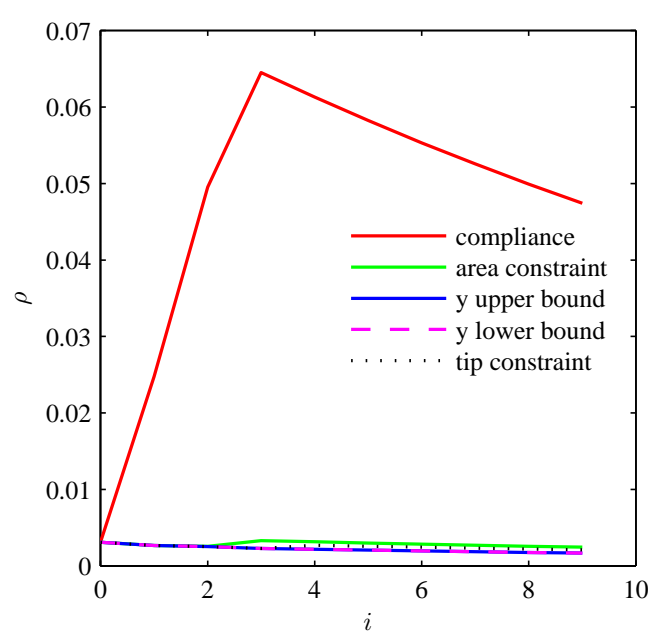

(f) Case 3

Figure 6. Convergence history of normalised function values (a), (c), (e), and corresponding damping terms (b), (d), (f) 


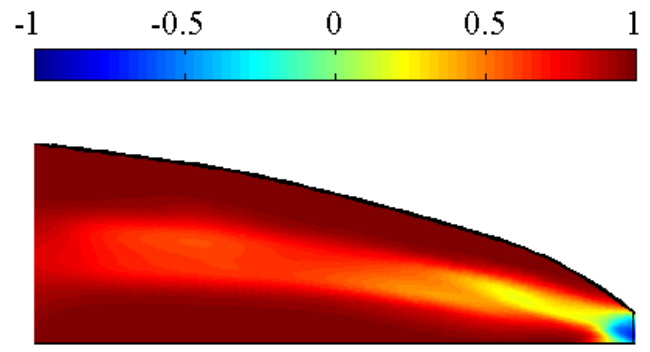

(a) $V_{1}$

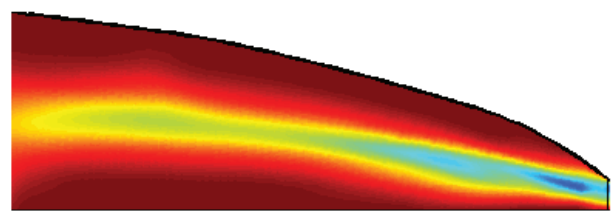

(b) $V_{3}$

Figure 7. In-plane lamination parameter distributions of the shape optimal variable stiffness plate using 34 by 10 design points

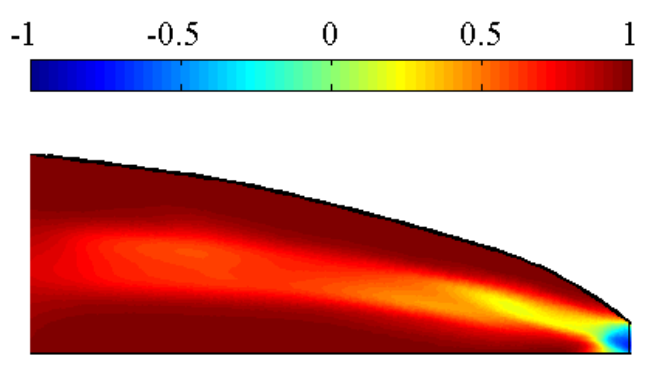

(a) $V_{1}$

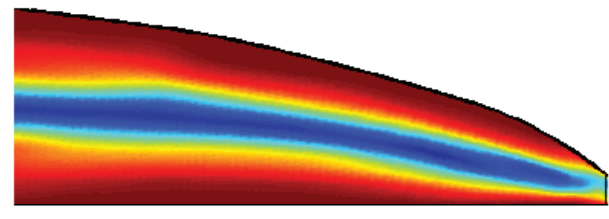

(b) $V_{3}$

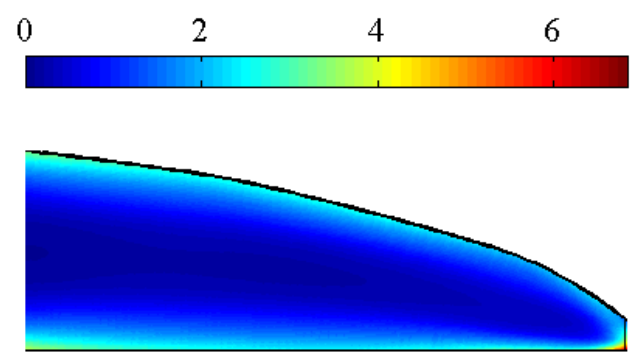

(c) $h / h_{0}$

Figure 8. In-plane lamination parameter and thickness distributions of the shape optimal variable stiffness and thickness plate using 34 by 10 design points and setting $h_{L}=0.1 \cdot h_{0}$ 